Journal of Mathematics and Informatics

Vol. 8, 2017, 57-68

ISSN: 2349-0632 (P), 2349-0640 (online)

Published 7 August 2017

www.researchmathsci.org

DOI: http://dx.doi.org/10.22457/jmi.v8a7

Journal of

Mathematics and

Informatics

\title{
Heuristic Neighbourhood Search Approach for Bulk
} Transportation Problem

\author{
P. Madhu Mohan Reddy ${ }^{1}$ and K.Chandrasekhar Reddy ${ }^{2}$ \\ ${ }^{1}$ Department of Mathematics, Siddharth Institute of Engineering \& Technology \\ Puttur-517583, Andhra Pradesh, India. \\ ${ }^{2}$ Department of Civil Engineering, Siddharth Institute of Engineering \& Technology \\ Puttur-517583, Andhra Pradesh, India. \\ e-mail: kamasani.kcr@gmail.com \\ Corresponding author. e-mail: mmrphdsv@gmail.com
}

Received 19 June 2017; accepted 1 August 2017

\begin{abstract}
The bulk transportation problems are to minimize the cost of carrying goods or people from sources to destinations using different types of transportation modes. In this paper, we studied a model of "heuristic neighborhood search approach for bulk transportation problem". We developed a heuristic algorithm for the bulk transportation problem for ' $m$ ' sources ' $n$ ' destinations. The process is illustrated in detail with the help of numerical example. Computer program for our proposed algorithm was developed in $\mathrm{C}$ language and results are reported. Our observations in these results are the CPU runtime is in micro seconds for higher values of the problems to obtain heuristic optimal solutions. We also compared the heuristic solution with optimal solution for different sizes of sources and destinations and found that the method is equally competent with optimal solution.
\end{abstract}

Keywords: Transportation, Source, Destination, Availability, Requirement, bulk cost.

AMS Mathematics Subject Classification (2010): 90B06

\section{Introduction}

Heuristic search approach is a more popular approach. Now a day's people are interested in quick and near optimal solution. Heuristic solution method is practically better than exact solution. When objective function is complicated, the exact solution method is difficult but heuristic search approach can attempt to solve. Heuristic search approach is equally and more popular than the exact approaches. If $\mathrm{n}$ value (size) is large exact solution method is complicated to solve but in heuristic search approach it is comfortable to solve.

It is true that common people and professionals frequently do not make decisions in the way prescribed by the mathematical models developed in operations research and management science. A simple decision model that people use is heuristics. For a long time heuristics were considered to be the second best to standard decision- theoretic tools such as linear models, networks, or classification and regression trees. 


\section{P. Madhu Mohan Reddy and K.Chandrasekhar Reddy}

In subsequent sections, we discussed about bulk transportation problem. We presented heuristic algorithm and illustrated with suitable numerical example and computational details are given.

\section{Bulk transportation problem}

Bulk transportation problem is a different kind of transportation problem. The problem of bulk transportation was first investigated by Maio and Roveda [1971] who presented an algorithm. Moreover, Murthy [1979], Babu et al. [2010] presented Lexi-Search algorithms and claimed that the effectiveness of this algorithm over branch and bound algorithm.

Junginer [1993] who proposed a set of logic problems to solve multi-index transportation problems have also conducted a detailed investigation regarding the characteristics of multi-index transportation problem model. Rautman et al. [1993], used multi-index transportation problem model to solve the shipping scheduling proposed that the employment of such transportation efficiency but also optimize the integral system. Hinojos [2000] investigated a multi-period two-echelon multi-commodity capacitated plant location problem, the problem deals with a facility location problem where one desires to establish facilities at two different distribution levels by selecting the time periods. Linda and Steef [2005] studied a multi-product lot-sizing model where, in any period, any portion of a reserved transportation capacity can be used in exchange for a guaranteed price. Purusotham and Murthy [2015] presented a multi-product bulk transportation problem to minimize the total cost of the bulk transportation. Latha [2013] presented three dimensional time minimization bulk transportation problem to minimize the total time of goods transportation. Guravaraju et al. [2015], Ellwein and Gray [1971] also studied different models on bulk transportation. problem.

In this paper, we developed heuristic algorithm for solving bulk transportation

\section{Problem description}

For heuristic bulk transportation problem, there are ' $m$ ' sources and ' $\mathrm{n}$ ' destinations. All destinations should get required capacity from sources. The ' $m$ ' sources should have capacity more than requirement of ' $\mathrm{n}$ ' destinations. The Cost/distance between sources and destinations are to be known and denoted by $\mathrm{C}(i, j)$. There is a restriction that each destination should fill the requirement from one source only. Usually the bulk cost is independent of the quantity of the products supplied. A source i can supply its product to a destination subjected to its availability and requirement of destinations. Heuristic approach is applied to get the solution. This solution is very near/ equal to the optimal solution. Usually it is less than ten per cent near to the in an optimal solution. The objective of the problem is to assign sources to the all destinations and minimize the total bulk transportation cost subjected to the availability and requirement constraints. The cost/distance of transportation of products from the sources to destinations is given.

In the bulk transportation problem $\mathrm{C}(\mathrm{i}, \mathrm{j})$ is the cost of transport of the requirement of the destination $\mathrm{DR}(\mathrm{j})$ from availability of source SA (i) and it is independent of the units of the products, hence it is called the bulk cost. Hence in the pattern $X(i, j)=0$ or 1 ; if it is 1 it means the source $i$ is supplying destination $j$, otherwise 0.i.e, $\mathrm{X}(\mathrm{i}, \mathrm{j})$ takes 0 or 1 values. There is a set of $S=\{1,2,3 \ldots m\}$ sources which 
Heuristic Neighbourhood Search Approach for Bulk Transportation Problem

produces a particular product and set of $\mathrm{D}=\{1,2,3 \ldots n\}$ destinations. The requirement of place $j \in D$ is DR (j) and the capacity of the source $i \in S$ is SA (i). The cost for bulk transportation from source $i$ to the place $j$ is $\mathrm{C}(\mathrm{i}, \mathrm{j}) \mathrm{i} \in \mathrm{S}$. $\mathrm{j} \in \mathrm{D}$. The objective is to assign sources to the destinations and minimize the total bulk transportation cost subjected to the availability and requirement constraints. The graphical representation of the problem is given in the following Figure-1. In the figure shapes of cylinder represent the sources and the boxes of cube shapes represent destinations.

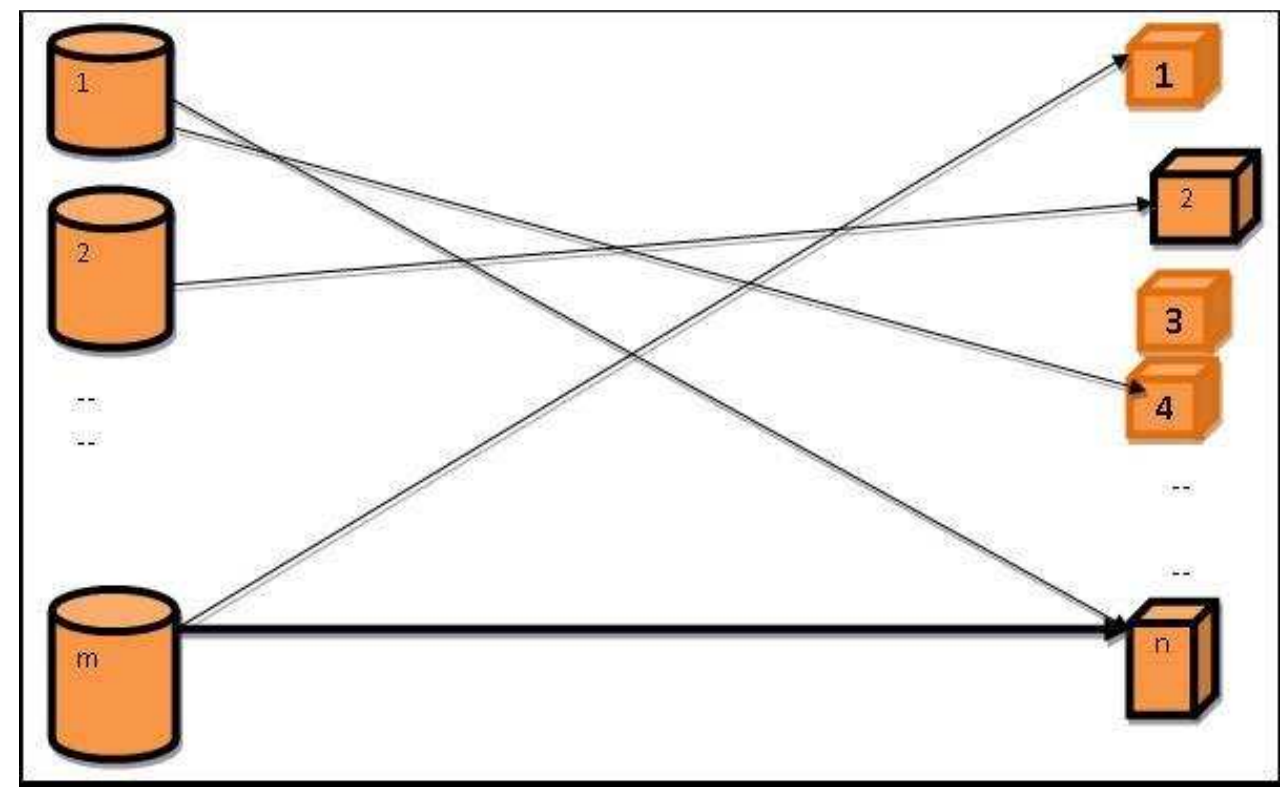

Figure 1:

The problem is discussed with a suitable numerical example. We proposed new algorithm using heuristic approach, which gives the solution less than ten per cent near to the optimal solution. The algorithm is tested and the computational results are also reported. This is a more generalized model and comes under combinatorial programming problems.

\section{Procedure for Heuristic bulk transportation problem}

The solution procedure for heuristic bulk assignment problem contains the following steps. Here different sources are assigning to different destinations.

\subsection{Procedure for initial feasible solution}

The steps of this procedure are same as the assignment problem and the addition is the necessary changes in the array SA and DR.

- ' $m$ ' sources, ' $\mathrm{n}$ ' destinations and the cost matrix $\mathrm{C}$ are given. Availability in Source is denoted by SA and Requirement in Destination is denoted by DR

- The first column of the cost matrix gives the various bulk costs of different sources assigned to the first destination. In this cost, we take the minimum cost 


\section{P. Madhu Mohan Reddy and K.Chandrasekhar Reddy}

and identify the corresponding source and assign the sources to the first destination and effective change in the source. The next, we search for the least cost among the destination in the second column with respect to the availability and fix for the second destination. This we continue till the lost destination is supplied. Finally all the $\mathrm{n}$ destinations are assigned to the sources and this given the Initial Feasible Solution. This is our initial feasible solution in the Heuristic algorithm.

\subsection{Search procedure}

In this procedure we always consider two pairs, i.e. $\alpha_{\mathrm{s}}$ source assigned to $\beta_{\mathrm{s}}$ destination and the cost is $\gamma_{\mathrm{s}}$. Another assignment (next) $\alpha_{\mathrm{s}+1}$ source assigned to $\beta_{\mathrm{s}+1}$ destination and the cost is $\gamma_{s+1}$. For this total cost is $\gamma_{s+} \gamma_{s+1}$ (say p). Now in both case we interchange the sources and destinations subjected to the condition the destination requirements are satisfied in the changed condition and find the new assignment cost. Let it be $\gamma_{\mathrm{s}}{ }^{1}$ and $\gamma_{\mathrm{s}+1}{ }^{1}$ and this total cost is $\gamma_{\mathrm{s}}{ }^{1}+\gamma_{\mathrm{s}+1}^{1}$ (say q). If $\gamma_{\mathrm{s}}+\gamma_{\mathrm{s}+1} \leq \gamma_{\mathrm{s}}{ }^{1}+\gamma_{\mathrm{s}+1}{ }^{1} \quad(\mathrm{p} \leq \mathrm{q})$, then interchange of the cost destinations will not reduce the total cost, so will not interchange. If $\gamma_{s+} \gamma_{s+1}>\gamma_{s}{ }^{1}$ $+\gamma_{s+1}^{1}(p>q)$ then this interchange cost will improve total cost and will effect the interchange. From this interchanges we will do in a systematic way till we reach the near optimal solution. If a particular source, destination not changed n-1 times then the process terminates and the corresponding assignment cost is the near optimal solution.

\section{Heuristic algorithm}

In this algorithm, we start with the above initial feasible solution (5.8.1). But we can also start with any randomly chosen feasible solution. In the feasible solution, we start with one pair of sets, where there are two destinations with two sources and corresponding costs. The first set destination can be random or here for our convenience, we start with the highest cost destination, the second set is the next pair of the assignment. [Note: whenever there is tie we can choose randomly]

Step 1. Consider the set $\alpha_{\mathrm{s}}$ to destination $\beta_{\mathrm{s}}$ with cost $\gamma_{\mathrm{s}}$ and also consider the next set $\alpha_{s+1}$ source to $\beta_{s+1}$ destination and cost $\gamma_{s+1}$ For this pair the cost is $\gamma_{s}+\gamma_{s+1}$ (p). Interchange for these two pairs i.e. $\alpha_{\mathrm{s}}$ source to $\beta_{\mathrm{s}+1}$ destination and $\alpha_{\mathrm{s}+1}$ source to $\beta_{\mathrm{s}}$ destination, let the corresponding cost be $\gamma_{s+}^{1} \gamma 1_{s+1=} q$.

If $p \leq q$ then with the above interchange of assignment there is no improvement in the total value. We always considered the feasibility of availability source and requirement of destination in the interchange. Hence this interchange is not affected. Go to Step -2 .

If $p>q$ then there will be improvement in solution (say (p-q)) and we will effect this interchange. Go to Step -2.

\section{Step 2.}

\section{(a) If $\mathbf{p} \leq \mathbf{q}$}

(i) If $\gamma_{\mathrm{s}} \geq \gamma_{\mathrm{s}+1}$ then $\left(\alpha_{\mathrm{s}}=\alpha_{\mathrm{s}}, \beta_{\mathrm{s}=} \beta_{\mathrm{s}}, \gamma_{\mathrm{s}=} \gamma_{\mathrm{s}}\right)$ and

$$
\left(\alpha_{\mathrm{s}+1=} \alpha_{\mathrm{s}+2}, \beta_{\mathrm{s}+1}=\beta_{\mathrm{s}+2}, \gamma_{\mathrm{s}+1=} \gamma_{\mathrm{s}+2}\right) \quad \text { go to Step-3 }
$$

(ii) If $\gamma_{\mathrm{s}}<\gamma_{\mathrm{s}+1}$ then $\left(\alpha_{\mathrm{s}=} \alpha_{\mathrm{s}+1}, \beta_{\mathrm{s}=} \beta_{\mathrm{s}+1}, \gamma_{\mathrm{s}=} \gamma_{\mathrm{s}+1}\right)$ and

$$
\left(\alpha_{\mathrm{s}+1}=\alpha_{\mathrm{s}+2}, \beta_{\mathrm{s}+1}=\beta_{\mathrm{s}+2}, \gamma_{\mathrm{s}+1}=\gamma_{\mathrm{s}+2}\right)
$$

[If $\mathrm{s}+2>\mathrm{n}$ then $\mathrm{s}+2=1$, i.e. the first set in the assignment is taken] go to Step-3. 
Heuristic Neighbourhood Search Approach for Bulk Transportation Problem

(b) If $\mathbf{p}>\mathbf{q}$ to improve the solution the interchange is effected, then the assignment costs are be $\gamma_{s}^{1}, \gamma l_{s+1}$.

(i) $\gamma_{\mathrm{s}}{ }^{1}<\gamma 1_{\mathrm{s}+1}$ Then $\left(\alpha_{\mathrm{s}=} \alpha_{\mathrm{s}+1}, \beta_{\mathrm{s}=} \beta_{\mathrm{s},}, \gamma_{\mathrm{s}}=\gamma 1_{\mathrm{s}+1}\right)$ and $\left(\alpha_{\mathrm{s}+1}=\alpha_{\mathrm{s}+2,}, \beta_{\mathrm{s}+1}=\beta_{\mathrm{s}+2,}, \gamma_{\mathrm{s}+1}=\gamma_{\mathrm{s}+2}\right)$ If $\left[\mathrm{DR}\left(\alpha_{\mathrm{s}}\right) \leq \mathrm{SA}^{1}\left(\beta_{\mathrm{s}+1}\right)\right.$ and DR $\left.\left(\alpha_{\mathrm{s}+1}\right) \leq \mathrm{SA}^{1}\left(\beta_{\mathrm{s}}\right)\right]$

Go to Step-3

Where, $\mathrm{SA}^{\mathrm{I}}(\mathrm{i})$ is the availability in the source after supply due to partial solution.

(ii) If $\gamma_{\mathrm{s}} \geq \gamma_{\mathrm{s}+1}$ then $\left(\alpha_{\mathrm{s}}=\alpha_{\mathrm{s}}, \beta_{\mathrm{s}=} \beta_{\mathrm{s}+1}, \gamma_{\mathrm{s}=} \gamma_{\mathrm{s}}\right)$ and $\left(\alpha_{\mathrm{s}+1=} \alpha_{\mathrm{s}+2}, \beta_{\mathrm{s}+1}=\beta_{\mathrm{s}+2}, \gamma_{\mathrm{s}+1=} \gamma_{\mathrm{s}+2}\right)$

If $\left[\mathrm{DR}\left(\alpha_{\mathrm{s}}\right) \leq \mathrm{SA}^{1}\left(\beta_{\mathrm{s}+1}\right)\right.$ and DR $\left.\left(\alpha_{\mathrm{s}+1}\right) \leq \mathrm{SA}^{1}\left(\beta_{\mathrm{s}}\right)\right]$ go to Step-3.

Step 3. In the first pair of assignment is not changing (n-1) times then the process is terminated. Go to step-4; otherwise go to Step-1.

Step 4. The final assignment schedule and corresponding cost is the heuristic optimal solution and stop.

Add the total costs of corresponding source and destination. Therefore this is ten per cent close to the optimal solution.

\section{Numerical illustration for heuristic bulk transportation}

For this bulk transportation problem concept and algorithm developed is illustrated by a numerical example for which the number of sources $m=4$ in set $S$, i.e. $S=\{1,2,3,4\}$ and the number of destinations $\mathrm{n}=9$ in set $\mathrm{D}$, i.e. $\mathrm{D}=\{1,2,3,4,5,6,7,8,9\}$. Each source has some availability and each destination has also requirement.

In the following numerical example, $\mathrm{C}(\mathrm{i}, \mathrm{j})$ is taken as non- negative integers it can be easily seen that this is not a necessary condition. The cost array $\mathrm{C}(\mathrm{i}, \mathrm{j})$ is given Table 1.

\begin{tabular}{|c|c|c|c|c|c|c|c|c|}
\hline 1 & 8 & 7 & 2 & 15 & 14 & 8 & 8 & 12 \\
\hline 9 & 13 & 6 & 1 & 14 & 3 & 15 & 13 & 3 \\
\hline 5 & 7 & 12 & 11 & 4 & 9 & 4 & 15 & 3 \\
\hline 10 & 7 & 6 & 6 & 10 & 11 & 12 & 5 & 14 \\
\hline
\end{tabular}

Table 1:

From the table- $1, C(2,6)=3$ means that the bulk cost of the $2^{\text {nd }}$ source is assigned to the $6^{\text {th }}$ destination is 3 .

Consider the source availability (SA) of the first source as 90 units, the second source as 100 units, the third source as 110 units and the fourth source having 100 units of availability. It is given in the Table 2 .

$\mathrm{SA}=$\begin{tabular}{|c|c|c|c|}
\hline 1 & 2 & 3 & 4 \\
\hline 90 & 100 & 110 & 100 \\
\hline \multicolumn{4}{|c|}{ Table 2: }
\end{tabular}




\section{P. Madhu Mohan Reddy and K.Chandrasekhar Reddy}

Suppose SA (3) = 110 means that the availability of source 3 is 110 units. The sum of the availability of 4 sources is $90+100+110+100=400$ units

Consider the Destinations of requirement (DR) is given in Table-3.

$\mathrm{DR}=$\begin{tabular}{|c|c|c|c|c|c|c|c|c|}
\hline 1 & 2 & 3 & 4 & 5 & 6 & 7 & 8 & 9 \\
\hline 50 & 30 & 20 & 30 & 20 & 40 & 40 & 40 & 30 \\
\hline
\end{tabular}

Table 3:

From the above Table- 3 , suppose DR (4) $=30$ units means that the requirement of destination 4 is 30 units. The sum of the requirement of all destinations $=50+30+20+30+20+40+40+40+30=300$ units.

Here, we can see that sum of the availability in four sources is 400 units and the sum of the requirement of 9 destinations is 300 units. Therefore, sum of the requirement of 9 destinations 300 units is less than the sum of the availability in four sources 400 units. This is a necessary condition for feasibility.

Our objective is to be finding minimum total cost supply to 9 destinations from the 4 sources.

\subsection{Initial feasible solution}

The initial feasible solution is assigned by the above procedure with respect to their requirement and availability. Therefore, initial feasible solution is given in the Table-4.

\begin{tabular}{|c|c|c|}
\hline S & D & C \\
\hline $\mathbf{1}$ & $\mathbf{1}$ & $\mathbf{1}$ \\
\hline $\mathbf{3}$ & $\mathbf{2}$ & $\mathbf{7}$ \\
\hline $\mathbf{2}$ & $\mathbf{3}$ & $\mathbf{6}$ \\
\hline $\mathbf{2}$ & 4 & $\mathbf{1}$ \\
\hline $\mathbf{3}$ & $\mathbf{5}$ & $\mathbf{4}$ \\
\hline $\mathbf{2}$ & $\mathbf{6}$ & $\mathbf{3}$ \\
\hline 3 & 7 & 4 \\
\hline 4 & 8 & 5 \\
\hline 1 & 9 & 12 \\
\hline
\end{tabular}

From the above table- 4 , the $1^{\text {st }}$ destination is assigned to the $1^{\text {st }}$ source, the $2^{\text {nd }}$ destination is assigned to the $3^{\text {rd }}$ source, the $3^{\text {rd }}$ destination is assigned to the $2^{\text {nd }}$ source, the $4^{\text {th }}$ destination is assigned to the $2^{\text {nd }}$ source, the $5^{\text {th }}$ destination is assigned to the $3^{\text {rd }}$ source, the $6^{\text {th }}$ destination is assigned to the $2^{\text {nd }}$ source, the $7^{\text {th }}$ destination is assigned to the $3^{\text {rd }}$ source, the $8^{\text {th }}$ destination is assigned to the $4^{\text {th }}$ source, the $9^{\text {th }}$ destination is assigned to the $1^{\text {st }}$ source.

The total cost of the initial feasible solution is $\mathrm{C}(1,1)+\mathrm{C}(3,2)+\mathrm{C}(2,3)+\mathrm{C}(2,4)+$ $\mathrm{C}(3,5)+\mathrm{C}(2,6)+\mathrm{C}(3,7)+\mathrm{C}(4,8)+\mathrm{C}(1,9)=1+7+6+1+4+3+4+5+12=43$.

The following Figure-2 represents the above initial feasible solution. The cylinder shapes represent sources and rectangle shapes represent destinations. The values in cylinder indicate sources and values in rectangles indicate destination. 
Heuristic Neighbourhood Search Approach for Bulk Transportation Problem

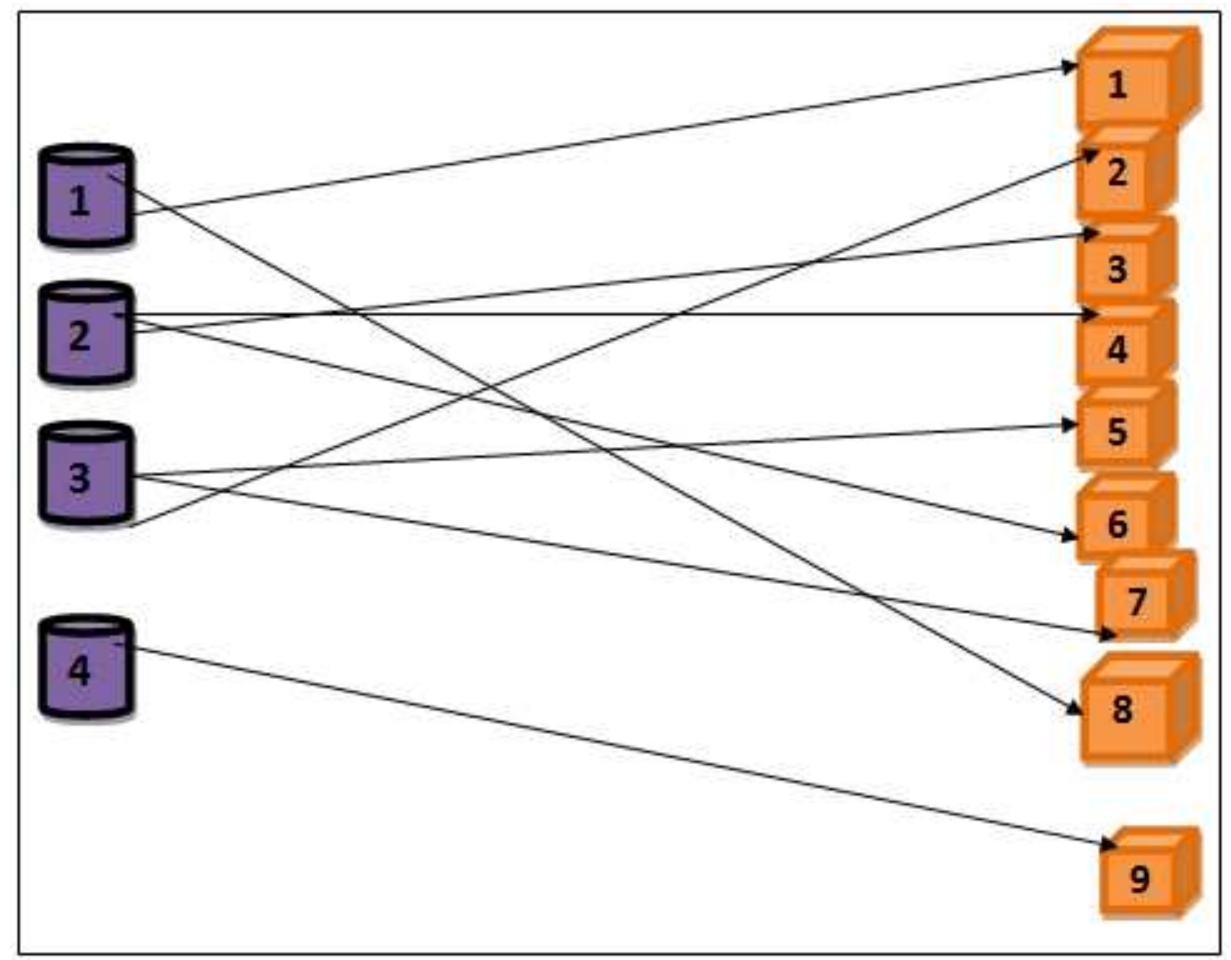

Figure 2:

The Figure -2 represents the initial solution. The source 1 is assigned to destinations 71 Source 2 is assigned to destinations $3,4,6$. Source 3 is assigned to destinations ? Source 4 is assigned to destination 8 and the sum of their corresponding costs are $\mathrm{C}(1,1)+\mathrm{C}(3,2) \mathrm{C}(2,3)+\mathrm{C}(2,4)+\mathrm{C}(3,5)+\mathrm{C}(2,6)+\mathrm{C}(3,7)+\mathrm{C}(4,8)+\mathrm{C}(1,9)$

$=1+7+6+1+4+3+4+5+12=43$.

According to the pattern represented in the Figure-5 is satisfies the 9 destinations requirement from 4 sources. After the supply the availability in source 1 is (90-50-40) 0 , availability in source 2 is (100-20-30-40) 20, availability in source 3 is (110-30-20-40) 20 , availability in source 4 is $(100-40) 60$. i.e. $\mathrm{SA}^{1}(4)=60$.

\subsection{Search table}

From the Table-5 the columns S, D and C represent Sources, Destinations and Costs respectively. The destinations are represented under column $\mathrm{D}$ as 1, 2, 3...9, corresponding sources are under column $\mathrm{S}$ and corresponding costs under column C. As a result $(3,2) \rightarrow 7$ is a set where the $3^{\text {rd }}$ source is assigned to the $2^{\text {nd }}$ destination with cost 7 .

In the heuristic algorithm, first we consider one set of sources, destinations and cost. Take the next one as the second set. In the algorithm we consider two sets of sources to destinations and interchange to reduce the total assignment cost under the consideration. For convenience, we select the set $(1,9) \rightarrow 12$, where source 1 is assigned 


\section{P. Madhu Mohan Reddy and K.Chandrasekhar Reddy}

to the $9^{\text {th }}$ destination with the cost 12 , which is the highest cost in the initial assignment and we are taking this as the first set.

\begin{tabular}{|c|c|c|c|c|}
\hline 1 & $\mathbf{S}$ & D & C & 1 \\
\hline- & 1 & 1 & 1 & - \\
\hline 1 & 3 & 2 & 7 & 8 \\
\hline- & 2 & 3 & 6 & - \\
\hline - & 2 & 4 & 1 & - \\
\hline - & 3 & 5 & 4 & - \\
\hline - & 2 & 6 & 3 & - \\
\hline - & 3 & 7 & 4 & - \\
\hline - & 4 & 8 & 5 & - \\
\hline \multirow[t]{2}{*}{3} & 1 & 9 & 12 & 3 \\
\hline & & & 43 & 8 \\
\hline
\end{tabular}

Table 5:

$\left(\alpha_{\mathrm{s}=1} 1, \beta_{\mathrm{s}=9}, \gamma_{\mathrm{s}=12}\right)$ i.e. this is the first set in the pair to consider.

For the second set, we consider the next pair of the first set in the assignment i.e. $(1,1) \rightarrow 1$, where source 1 is assigned to the $1^{\text {st }}$ destination with the cost 1 , because the first set $(1,9) \rightarrow 12$ is the lost in the assignment and the next one is the $1^{\text {st }}$ one i.e. $(1,1) \rightarrow 1$. Hence

Let $\quad\left(\alpha_{s+1=1}, \beta_{s+1}=1, \gamma_{s+1}=1\right)$ i.e. this is the second set in pair.

So the two sets are

$$
\begin{aligned}
& \left(\alpha_{\mathrm{s}=1}, \quad \beta_{\mathrm{s}=} 9, \gamma_{\mathrm{s}=} 12\right) \\
& \left(\alpha_{\mathrm{s}+1=1}, \beta_{\mathrm{s}+1}=1, \gamma_{\mathrm{s}+1=} 1\right)
\end{aligned}
$$

Step 1: $\gamma_{\mathrm{s}}+\gamma_{\mathrm{s}+1=} 12+1=13=\mathrm{p}$.

Now, we interchange the in pairs $\alpha_{\mathrm{s}}$ and $\alpha_{\mathrm{s}+1}$ then corresponding costs are also changed and let the corresponding costs for the pairs be are $\gamma_{\mathrm{s}}{ }^{1}$ and $\gamma 1_{\mathrm{s}+1}$. There the above two pairs becomes

Step 2: (b) $\quad \mathbf{p}=\mathbf{q}$ i.e., $13=13$.

$$
\begin{aligned}
& \left(\alpha_{\mathrm{s}=1}, \beta_{\mathrm{s}+1}=2, \gamma_{\mathrm{s}}=8\right) \\
& \left(\alpha_{\mathrm{s}+1}=1, \beta_{\mathrm{s}=9}, \gamma 1_{\mathrm{s}+1}=3\right) \\
& \gamma_{\mathrm{s}}{ }^{1}+\gamma 1_{\mathrm{s}+1}=12+1=13=\mathrm{q} \quad \text { go to Step } 2
\end{aligned}
$$

This interchange will not improve the solution. Hence do not effect the change

(i) Here the first set is $\left(\alpha_{\mathrm{s}=} 1, \beta_{\mathrm{s}=} 9, \gamma_{\mathrm{s}=} 12\right)$

For the second set we consider

$(3,2) \rightarrow 7$, where source 3 is assigned to the $2^{\text {nd }}$ destination with cost 7 , hence let $\left(\alpha_{s+1=3}, \beta_{s+1=}, \gamma_{s+1}=7\right)$ i.e. this is the second set in pair.

Here $\mathrm{SA}^{1}=\{0,20,20,60\}$

So the two sets are 
Heuristic Neighbourhood Search Approach for Bulk Transportation Problem

$\left(\alpha_{\mathrm{s}=1}, \beta_{\mathrm{s}=} 9, \gamma_{\mathrm{s}=} 12\right)$

$\left(\alpha_{s+1}=3, \beta_{s+1}=2, \gamma_{s+1}=7\right)$, here $\operatorname{DR}(9)=30, \operatorname{DR}(2)=30$ Go to Step 1 .

Step 1: $\gamma_{\mathrm{s}}+\gamma_{\mathrm{s}+1}=12+7=19=\mathrm{p}$

Now, we interchange two pairs $\alpha_{\mathrm{s}}$ and $\alpha_{\mathrm{s}+1}$ then corresponding costs could also be changed and let the corresponding costs be the two pairs are $\gamma_{s}{ }^{1}$ and $\gamma l_{s+1}$. Therefore, the above two pairs becomes

$$
\begin{aligned}
& \left(\alpha_{\mathrm{s}=} 3, \beta_{\mathrm{s}=} 9, \gamma_{\mathrm{s}}{ }^{1}=12\right) \\
& \left(\alpha_{\mathrm{s}+1=1}, \beta_{\mathrm{s}+1}=2, \gamma 1_{\mathrm{s}+1=7}=7\right) \\
& \gamma_{\mathrm{s}}{ }^{+}+\gamma 1_{\mathrm{s}+1}=3+8=11=\mathrm{q}
\end{aligned}
$$

Here $\mathrm{SA}^{1}=\{0,20,20,60\}$ Go to step 2 .

Step 2: (b) p>q i.e., $19>11$

This interchange will improve the total solution. Hence, we have to effect the change. p$\mathrm{q}=19-11=8$ is reduced from the total value.

In this process, we notice that requirement of the $9^{\text {th }}$ destination is supplied from the $3^{\text {rd }}$ source and requirement of the $2^{\text {nd }}$ destination is supplied from the $1^{\text {st }}$ source. The $3^{\text {rd }}$ and the $1^{\text {st }}$ source come under the column 1 left of D and corresponding costs 3 and 8 comes under column 1 right of the column $\mathrm{D}$ in the search table, Table- 8 . The reduced cost $\mathrm{p}-\mathrm{q}=8$ is at the right side $\mathrm{D}$ in the lost row. Go to Step 1

In the algorithm the set $(1,2) \rightarrow 8$ where interchanged with all the (n-1=8) sets, it is not interchanged, therefore the algorithm is terminated.

At the end of the search, the current value and heuristic optimal cost is 35 (43-8) and it is ten per cent close to the optimal solution. There is only 1 interchange in the algorithm for the problem for getting the near optimal solution. The change of destinations in the $\mathrm{i}^{\text {th }}$ interchange is given in the $\mathrm{i}^{\text {th }}$ column of the left of $\mathrm{D}$ and change of costs in the $\mathrm{i}^{\text {th }}$ column of the right of $\mathrm{D}$. The 9 destinations get the supply of their requirements from 4 sources and the corresponding order pairs are

$(1,1),(3,2),(2,3)(2,4),(3,5),(2,6),(3,7),(4,8),(1,9)$.

The sum of corresponding costs is

$\mathrm{C}(1,1)+\mathrm{C}(3,2)+\mathrm{C}(2,3)+\mathrm{C}(2,4)+\mathrm{C}(3,5)+\mathrm{C}(2,6)+\mathrm{C}(3,7)+\mathrm{C}(4,8)+\mathrm{C}(3,9)$

$=1+8+6+1+4+3+4+5+3=35$ and this is called as improved feasible solution, it coincides with the exact solution.

The following Figure-3 represents an initial feasible solution. The cylinder shapes represent sources and rectangle shapes represent destinations. The values in cylinder indicate sources and values in rectangles indicate destinations.

The above Figure-3 represents the initial solution. The source1 is assigned to destinations 1,9. Source 2 is assigned to destinations 3, 4, 8. Source 3 is assigned to destinations 1, 6 Source 4 is assigned to destinations 2,9 and the sum of their corresponding cost are $\mathrm{C}(1,1)+\mathrm{C}(1,2)+\mathrm{C}(2,3)+\mathrm{C}(2,4)+\mathrm{C}(3,5)+\mathrm{C}(2,6)+\mathrm{C}(3,7)+$ $\mathrm{C}(4,8)+\mathrm{C}(3,9)=1+3+6+1+3+8+4+4+5=35$. 
P. Madhu Mohan Reddy and K.Chandrasekhar Reddy

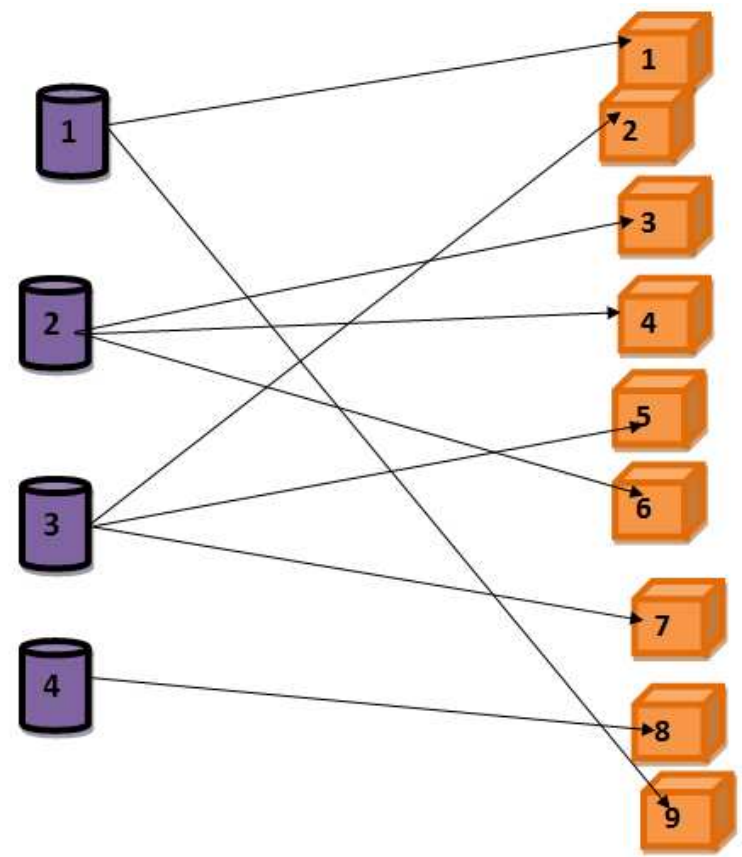

Figure 3:

According to the pattern represented in Figure-3 satisfies the 9 destinations of their requirements from 4 sources.

\section{Experimental results heuristic bulk transportation problem}

We write computer program for this algorithm in $\mathrm{C}$ language and it is verified by the system COMPAQ dx2280 MT. We tried a set of problems for different sizes of S and D. The cost matrix $C(i, j)$ takes the values uniformly random in $[0,100]$. The results tabulated in the Table- 6 .

\begin{tabular}{|c|c|c|c|c|c|}
\hline \multirow{2}{*}{ SN } & \multicolumn{2}{|c|}{ Problem dimension } & \multirow{2}{*}{ NPT } & \multicolumn{2}{c|}{ CPU run time in seconds } \\
\cline { 2 - 5 } & S & D & & Avg.IT & Avg.ST \\
\hline 1 & 5 & 8 & 5 & 0 & 0 \\
\hline 2 & 10 & 15 & 5 & 0 & 0 \\
\hline 3 & 15 & 20 & 5 & 0 & 0 \\
\hline 4 & 20 & 25 & 5 & 0 & 0 \\
\hline 5 & 25 & 30 & 5 & 0 & 0 \\
\hline 6 & 30 & 35 & 5 & 0 & 0 \\
\hline 7 & 35 & 40 & 5 & 0 & 0 \\
\hline 8 & 40 & 45 & 5 & 0 & 0 \\
\hline
\end{tabular}


Heuristic Neighbourhood Search Approach for Bulk Transportation Problem

\begin{tabular}{|c|c|c|c|c|c|}
\hline 9 & 45 & 50 & 5 & 0 & 0 \\
\hline 10 & 50 & 60 & 5 & 0 & 0 \\
\hline 11 & 55 & 65 & 5 & 0 & 0 \\
\hline 12 & 60 & 75 & 5 & 0 & 0 \\
\hline 13 & 65 & 80 & 5 & 0 & 0 \\
\hline 14 & 70 & 85 & 5 & 0 & 0 \\
\hline
\end{tabular}

Table 6:

In the above table- 6 the notations represent $S=$ Number of sources, $D=$ Number of destinations, NPT $=$ Number of problems tried, Avg.IT $=$ Average CPU run time to obtain an initial solution. Avg.ST = Average CPU run time to obtain the feasible solution. For each instance five data sets are tested. It is seen that time required for the search of the near optimal solution is 0 seconds.

\section{Comparison for heuristic bulk transportation solution and optimal solution}

We implement the proposed heuristic algorithm with $\mathrm{C}$ language for this model. We tested the proposed algorithm by different set of problems and compared the heuristic optimal solution with exact solutions (got by lexi- search algorithm).

The Table-7 shows that the comparative results of different sizes of S and D.

\begin{tabular}{|c|c|c|c|c|c|c|}
\hline \multirow{2}{*}{ SN } & \multicolumn{2}{|c|}{$\begin{array}{c}\text { Problem } \\
\text { dimension }\end{array}$} & \multicolumn{2}{c|}{$\begin{array}{c}\text { CPU run time in } \\
\text { seconds }\end{array}$} & $\begin{array}{c}\text { Heuristic } \\
\text { solution }\end{array}$ & $\begin{array}{c}\text { Optimal } \\
\text { solution }\end{array}$ \\
\cline { 2 - 5 } & S & D & IT & ST & & 7 \\
\hline 1 & 5 & 7 & 0 & 0 & 7 & 13 \\
\hline 2 & 10 & 13 & 0 & 0 & 14 & 40 \\
\hline 3 & 15 & 20 & 0 & 0 & 40 & 53 \\
\hline 4 & 20 & 25 & 0 & 0 & 54 & 90 \\
\hline 5 & 25 & 30 & 0 & 0 & 90 & 112 \\
\hline 6 & 30 & 35 & 0 & 0 & 114 & \\
\hline
\end{tabular}

Table 7:

For the above six sets of problems in Table-7, we calculated both heuristic optimal solutions and exact solutions (by lexi search algorithm). We find almost equal values in both the cases.

\section{Conclusion}

In this paper, we studied a model of "heuristic neighborhood search approach for bulk transportation problem". We developed a heuristic algorithm for the bulk transportation problem for ' $m$ ' sources ' $n$ ' destinations. The process is illustrated in detail with the help 
P. Madhu Mohan Reddy and K.Chandrasekhar Reddy

of numerical example of size with ' 4 ' sources and ' 9 ' destinations. We got initial solution is 43 but heuristic solution is 35 for this example. Computer program for our proposed algorithm was developed in $\mathrm{C}$ language and results are reported. Our observations in these results are the CPU runtime is in micro seconds for higher values of the problems to obtain heuristic optimal solutions. We also compared the heuristic solution with optimal solution for six different sizes of source and destinations and found that the method is equally competent with optimal solution.

\section{REFERENCES}

1. L.B.Ellwein and P.Gray, Solving fixed charge location - allocation problems with capacity and configuration constraints, AIIE Transactions, 3 (1971) 290-298.

2. P.Guravarajulu, S.Purusotham, C.Suresh Babu and P.Madhu Mohan Reddy, Twostage multi objective variant bulk TP model, International Journal of Advanced Research in Computer Science and Software Engineering, 59 (2015) 115-125.

3. Y.Hinojosa, A multi-period two-echelon multi-commodity capacitated plant location problem, European Journal of Operational Research, 123 (2000) 271 -291.

4. W.Junginer, On representatives of multi-index transportation problems, European Journal of Operational Research, 66 (1993) 353-371.

5. N.Linda Van and V.Steef Van De, Multi-product lot-sizing with a transportation capacity reservation contract, European Journal of Operational Research, 165 (2005) $127-138$.

6. A.Maio and C.Roveda, An all zero-one algorithm for a certain class of transportation problems, Operations Research, 19 (1971) 1406-1418.

7. S.Purusotham and M.Sundara Murthy, An exact algorithm for multi - product bulk transportation model, International Journal of Advanced Research in Computer Science and Software Engineering, 5 (2015) 115-125.

8. C.A.Rautman, Reid and E.E.Ryder, Scheduling the disposal of nuclear waste material in a geologic repository using the transportation model, Operations Research, 41 (1993) 459-469.

9. K.Sobhan Babu and M.Sundara Murthy, An efficient Algorithm for variant bulk transportation problem, International Journal of Engineering Science and Technology, 2 (2010) 2595-2600.

10. M.Sundara Murthy, A bulk transportation problem, Opsearch,13 (1979) 143-155.

11. A.Vidhyul Latha, Three dimensional time minimization bulk transportation problem, International Journal of Research in Mathematics \& Computation, 1 (2013) 26-40. 